Studierenden, die bei ihm das Vertiefungsgebiet „klientenzentrierte Gesprächsführung“ belegt haben; sie dokumentieren damit auch erste Versuche, selbstständig Beratung anzubieten. Positiv fällt auf, dass auch offen über typische Anfängerschwierigkeiten gesprochen wird. Hierzu gehören: die Identifikation mit den Betroffenen, geringe Einfühlung, zu starke Lenkung oder die Verweigerung von Verantwortung für den Beratungsprozess. Gerade diese realitätsbezogene Darstellung kann die Lernenden zum Weitermachen ermutigen, auch wenn sich anfänglich Schwierigkeiten auftun. Ich meine, dass gerade typische Anfängerfehler, die während der Ausbildungszeit gut supervidiert und aufgearbeitet werden, für die weitere berufliche Entwicklung sehr fruchtbar sein können. Denn diese „Fehler“ braucht man nicht mehr zu wiederholen. Kolleginnen und Kollegen, die Gesprächsführung nicht nur praktisch vorführen, sondern auch theoretisch unterrichten, werden sich über die vielen übersichtlichen Tabellen freuen. Diese kann man nämlich gut in der Lehre als Folie verwenden. Nando Belardi, Chemnitz

\section{Die Themenschwerpunkte der nächsten Hefte}

Heft 2/2001 Coaching für Frauen

Heft 3/2001 Beziehung in Supervision und Coaching

Heft 4/2001 Zur Differenz von Führen und Beraten

Heft 1/2002 Gruppenphänomene in Supervision, Coaching, Organisationsberatung

Heft 2/2002 Evaluation

\section{Pressemitteilung}

\section{ANSE startete ein Webforum für professionelle Diskussion und Austausch von Informationen zur Supervision}

Seit dem 7. Januar 2001 bietet ANSE (Association of national organizations for supervision in Europe) Supervisoren weltweit die Möglichkeit, ein einzigartiges Forum für kollegiale Diskussionen und für den Austausch von Informationen zu nutzen. Auf diesem ersten weltweiten Webforum für Supervision kann jede/r Supervisor/in in der von ihm/ihr bevorzugten Sprache

- Diskussionen eröffnen oder fortsetzen über Themen zur Supervision, Coaching, Teamentwicklung, Organisationsentwicklung etc.,

- Informationen präsentieren, die für Kollegen relevant sein könnten,

- Fragen an Kollegen stellen.

Wir hoffen, daß diese Initiative auf ein Bedürfnis nach Austausch von Informationen und Diskussionen zu professionellen Themen treffen wird und einen Beitrag zur Entwicklung der Profession liefern kann. Außer diesem Forum bietet die ANSE Webseite Informationen über ANSE, über ihre Mitgliedsorganisationen und Links zu den jeweiligen Supervisionsverbänden und zu Informationen über Supervision sowie ein Lexikon der Fachbegriffe in neun Sprachen.

Drs. Louis van Kessel Präsident der ANSE

P.S.: Gestalten Sie bitte eine LINK von Ihrer Webseite zu den Webseite der ANSE.

http://www.supervision-eu.org/anse 